\title{
Dynamic Modeling and Parametric Analysis of the Magnetic Stiffness on a Radial Heteropolar Rotor Magnetic Bearing (RMB)
}

\author{
Rodrigo Trentini ${ }^{1{ }^{1 *},}$ Daniel dos Santos ${ }^{1}$, Oscar Reichow ${ }^{1}$, Rodrigo Piontkewicz ${ }^{1}$ \\ Federal Institute of Santa Catarina - IFSC \\ Campus Jaragu'a do Sul - Rau, Jaragua' do Sul, Brazil \\ Email: rodrigo.trentini@ifsc.edu.br \\ * Corresponding author
}

\begin{abstract}
This paper presents both the dynamic modelling and the study of the variation of certain physical parameters (pole area, gap and base current) that change the magnetic stiffness of a radial heteropolar Rotor Magnetic Bearing (RMB) aiming at the analysis of the magnitude of its control current for three different equilibrium points. The RMB is modelled as a fully uncoupled symmetric active 8-pole magnetic bearing. The analysis is performed using spectral cubes for a better visualisation of the posed problem. The saturation analysis of the RMB is also performed. At last, it is shown that a control current with the same magnitude as the base current is reached with minimum pole area and air gap $s_{0}=528 \mu \mathrm{m}$.
\end{abstract}

Index Terms-Rotor Magnetic Bearing, Magnetic Stiffness, Sensitivity.

\section{INTRODUCTION}

Most electric machines make use of conventional mechanical bearings. They are based on rolling or hydrostatic oil elements characterised by the presence of friction during their operation [1]. Thus, losses of torque, heating and the so-called dead zone occur due to static friction, which imposes a barrier to the development of more efficient engines, in addition to physically limiting the delivery of high speeds to the load. Particularly in the case of the dead zone, this non-linearity especially affects applications of torque control at zero speed, as in the area of Robotics [2].

In some special applications, such as extreme vacuum generation, explosive atmospheres, vibration control, at high speeds and torque control, magnetic suspension systems applied to bearings become viable [3]. The main reason, in addition to eliminating the frictional forces and mechanical contact of the assembly, is that these bearings reduce its wear, prolonging the service life and, consequently, reducing maintenance interventions. Moreover, they are considered environment friendly, as they do not require the use of oil for their lubrication [4].

Rotor Magnetic Bearings (RMBs) are cited whenever the magnetic bearing application is related to electrical machines. For such applications, the lack of mechanical contact (friction) is very welcome mostly when high speeds are required.

There are several possible types and configurations for RMBs, from passive - which are composed only of permanent magnets - to the most sophisticated active magnetic bearings
(AMBs) - which have electromagnets controlled by microcontrolled systems. There are also hybrid magnetic bearings, which join permanent magnets with electromagnets.

The study of RMBs, non-linear and naturally unstable systems, acquires high technological importance. The vast majority of current industrial magnetic levitation applications use AMBs. For example, the Norwegian company Statoil implemented the first subsea gas compression system in the Asgard gas field to use magnetic bearings in order to extend the life of its compressors [5]. The German company Siemens delivered the first oil-free steam turbine to lubricate its bearings at the end of 2010. Unlike other steam turbines, it operates at a relatively high speed $(5,700 \mathrm{rpm})$ without the need for oil cooling [6]. The University of Texas has developed a flywheel for a bus that operates at 40,000 rpm with magnetic bearings. The purpose of the prototype is to increase the useful life of one of the most sensitive and required systems in the public transport fleet [7].

The physical construction of an RMB is crucial for its further control, given that its parameters might affect the socalled magnetic stiffness, which is a measure of the required current to maintain the rotor at a certain equilibrium position. Thus, depending upon the magnetic stiffness, the RMB control system might be more or less sensitive to disturbance, parameter variations and noise.

For instance, Liu et al. [8] analyse the effects of the rotational speed and phase compensation angle on the unbalanced vibration control on a RMB. It is found that the synchronous control currents would increase rapidly with the increase of rotational speed if the unbalance vibration cannot be controlled. The authors use a PID controller with a notch filter to reduce the unbalance vibration response of the rotor system. The effects of the rotational speed and phase compensation angle on the unbalanced vibration control are analysed in detail.

Yet within the control field, Zheng et al. [9] show that the proposed control method have significant control effect on the periodic vibrations produced by the unbalanced force of a RMB. The authors use a novel structure for an Iterative Learning Controller (ILC). The research achievements can provide theoretical references and experimental basis for the 
further application of AMB in the high-precision and highspeed fields.

With this information in mind, this work analyses the influence of three critical parameters on the magnetic stiffness of a RMB, namely the pole area, the air gap and the base current. The study is performed to give the designer a visual plot of the magnitude of RMB's control current for variations in the cited parameters, since that analysis for such a complex nonlinear function is not mathematically trivial.

The RMB is regarded as a fully uncoupled symmetric active 8-pole magnetic bearing. Despite being one of the most basic configurations, there is still recent control development [10] and it is also used as a study base for newer configurations [11].

Its modeling is presented in Sec. II. Section III deals with the effects of the magnetic stiffness on the control current, whilst the analysis of its results is performed in Sec. IV. Section $\mathrm{V}$ presents the conclusions of the paper.

\section{Modelling OF A RAdial 8-POLE RMB}

An 8-pole RMB can be understood as a set of 4 magnetic attractors, and each is formed by a magnetic circuit as shown in Fig. 1.

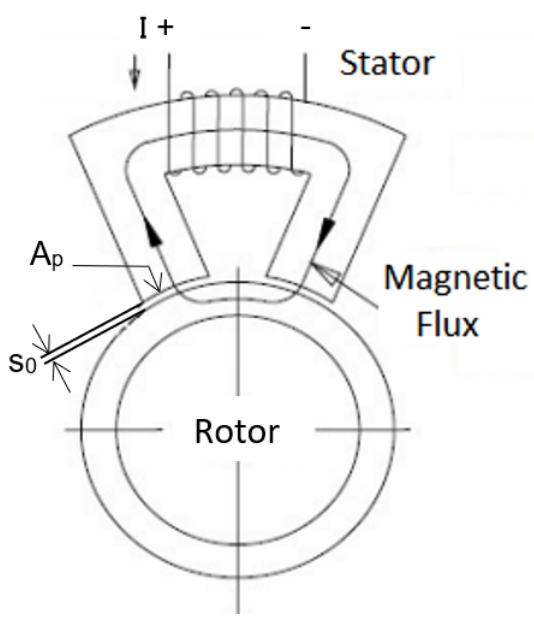

Fig. 1. Representation of magnetic attractor [12]

Acting in opposite pairs to the axis, attractors can control the position of the shaft with an independent signal for each pair and for this reason, this bearing has as main advantages its symmetry and uncoupled control action. Its picture and schematics are shown in Fig. 2.

The magnetic force $f$, written as a function of the air gap distance, can be compared to the force of a simple magnetic levitator, but in this case the constant $k$ is determined analytically [14]. Thus,

$$
f=\frac{1}{4} \mu_{0} N^{2} A_{p}\left(\frac{i}{s_{0}}\right)^{2}=k\left(\frac{i}{s_{0}}\right)^{2},
$$

with $\mu_{0}, N, A_{p}, i$ and $s_{0}$ being, respectively, the air permeability, number of turns, pole area, electric current and air gap.

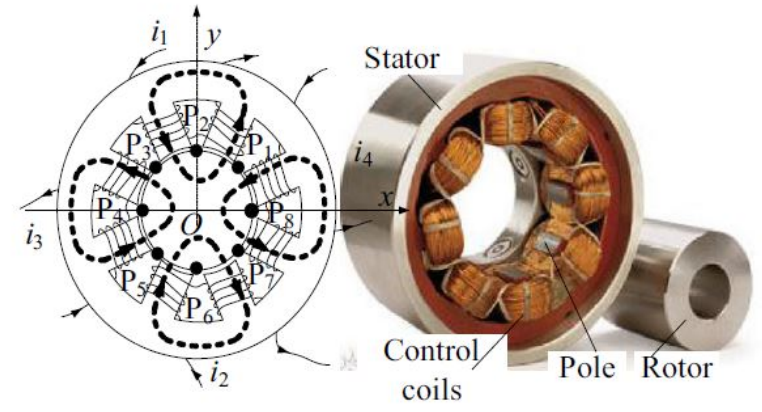

Fig. 2. Representation of an 8-pole RMB [13].

When applying this model to a pair of poles of an RMB, there is a change in the expression of the magnetic force, given that the magnetic force is effective only in its projections parallel to the displacement of the $x$-axis. Therefore, the expression of the magnetic force for a pair of poles of a magnetic bearing is,

$$
f=\frac{1}{4} \mu_{0} N^{2} A_{p}\left(\frac{i}{s_{0}}\right)^{2} \cos \alpha_{p}
$$

being $\alpha_{p}$ the angle between the normal of the gap area and the axis of symmetry of the magnetic attractor.

However, 8-pole RMB work usually in the differential mode. A base current $i_{0}$ that corresponds to the current in the bearing equilibrium condition is established. Hence, the control current corresponds to the control action in an opposite way over the base current.

This configuration allows to generate forces in both directions, in addition to improving linearity due to symmetry. It also allows the coefficients of the linearized system to be freely established, since $i_{0}$ no longer depends on the weight of the body to be levitated. Fig. 3 shows the implementation of the cited differential mode, with $i_{x}$ being the control current.

Separating the windings from the base and control currents allows a number of turns of the base winding $\omega_{0}$ to be implemented other than the number of turns of the controlled winding $\omega_{x}$. This also allows the use of wires with different cross-sectional areas. The magnetic force in the $x$-axis becomes,

$$
f=\frac{1}{4} \mu_{0} A_{p} \cos \alpha_{p}\left[\frac{\left(\omega_{0} i_{0}+\omega_{x} i_{x}\right)^{2}}{\left(s_{0}-x \cos \alpha_{p}\right)^{2}}-\frac{\left(\omega_{0} i_{0}-\omega_{x} i_{x}\right)^{2}}{\left(s_{0}+x \cos \alpha_{p}\right)^{2}}\right]_{(3)}
$$

which linearized turns to,

$$
f \approx \underbrace{\mu_{0} A_{p} \cos \alpha_{p}^{2} \frac{\left(\omega_{0} i_{0}\right)^{2}}{\left(s_{0}\right)^{3}}}_{k_{s}} x+\underbrace{\mu_{0} A_{p} \cos \alpha_{p} \frac{\left(\omega_{0} \omega_{x} i_{0}\right)}{\left(s_{0}\right)^{2}}}_{k_{i}} i_{x} .
$$




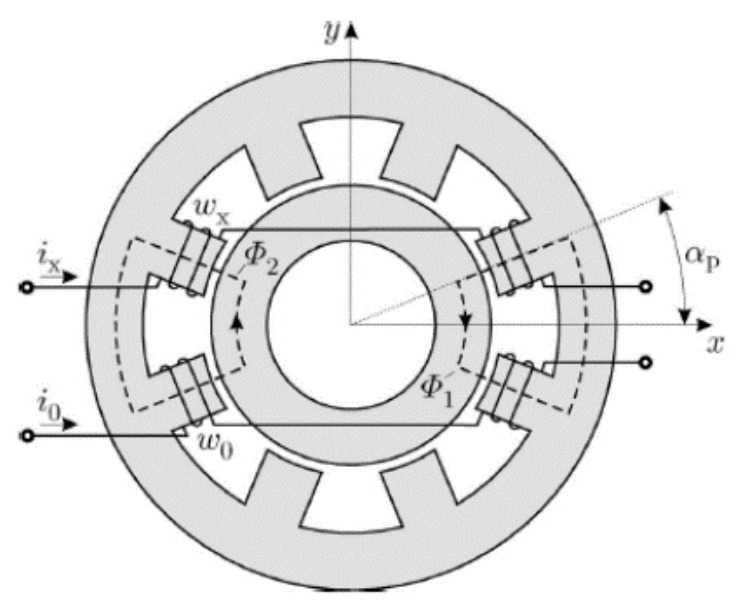

Fig. 3. Differential actuator with segregated base current [14]

For the dynamic system, a direct approach is to consider the rotor-axis model as a rigid system as in Fig. 4.

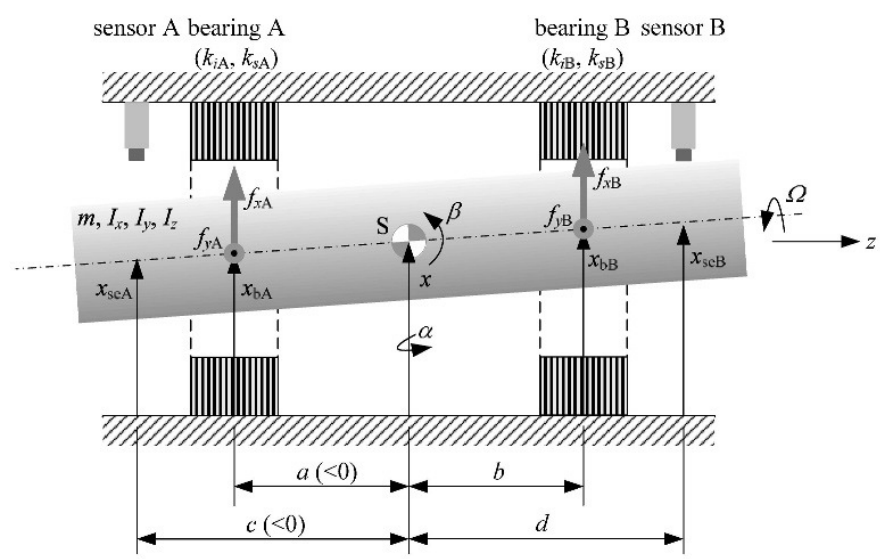

Fig. 4. Rigid rotor with sensors and bearings [15].

For simplicity, axial movement is omitted as it can be considered decoupled. Hence, there are four degrees of freedom (DOF): the small displacements of the centre of mass $x$ and $y$ and the Euler angles $\alpha$ and $\beta$, which are combined in the state vector $q=[\beta, x,-\alpha, y]^{T}$. The displacements measured by the sensors are included in the output vector $y=\left[x_{s e A}, x_{s e B}, y_{s e A}, y_{s e B}\right]^{T}$. The vector of external forces is better described as vertical and horizontal components in the bearing positions to include the magnetic forces and given by $u_{f}=\left[f_{x A}, f_{x B}, f_{y A}, f_{y B}\right]^{T}$. The rotor dynamics is then given by,

$$
\begin{aligned}
M \ddot{q}+G \dot{q} & =B u_{f}, \\
y & =C q .
\end{aligned}
$$

Matrices $M$ (inertia), $G$ (gyroscopic), $B$ (input) and $C$ (output) are:

$$
\begin{aligned}
& M=\left[\begin{array}{cccc}
I_{f} & 0 & 0 & 0 \\
0 & m & 0 & 0 \\
0 & 0 & I_{x} & 0 \\
0 & 0 & 0 & m
\end{array}\right], \quad G=\left[\begin{array}{cccc}
0 & 0 & I_{z} \Omega & 0 \\
0 & 0 & 0 & 0 \\
-I_{z} \Omega & 0 & 0 & 0 \\
0 & 0 & 0 & 0
\end{array}\right], \\
& B=\left[\begin{array}{llll}
a & b & 0 & 0 \\
1 & 1 & 0 & 0 \\
0 & 0 & a & b \\
0 & 0 & 1 & 1
\end{array}\right], \quad C=\left[\begin{array}{llll}
c & 1 & 0 & 0 \\
d & 1 & 0 & 0 \\
0 & 0 & c & 1 \\
0 & 0 & d & 1
\end{array}\right] .
\end{aligned}
$$

However, instead of considering the magnetic forces due to $q$ displacements with the Euler angles, it is more appropriate to consider the vertical and horizontal displacements of the shaft at the bearing positions, with $q_{B}=\left[x_{b A}, x_{b B}, y_{b A}, y_{b B}\right]^{T}$.

Generalising the magnetic force in Eq. 4 for the rigid rotor gives,

$$
\begin{aligned}
& u_{f}=\left[\begin{array}{l}
f_{x A} \\
f_{x B} \\
f_{y A} \\
f_{y B}
\end{array}\right]=-\underbrace{\left[\begin{array}{cccc}
k_{s A} & 0 & 0 & 0 \\
0 & k_{s B} & 0 & 0 \\
0 & 0 & k_{s A} & 0 \\
0 & 0 & 0 & k_{s B}
\end{array}\right]}_{K_{s}}\left[\begin{array}{l}
x_{b A} \\
x_{b B} \\
y_{b A} \\
y_{b B}
\end{array}\right]+ \\
& \underbrace{\left[\begin{array}{cccc}
k_{i A} & 0 & 0 & 0 \\
0 & k_{i B} & 0 & 0 \\
0 & 0 & k_{i A} & 0 \\
0 & 0 & 0 & k_{i B}
\end{array}\right]}_{K_{i}}\left[\begin{array}{l}
i_{x A} \\
i_{x B} \\
i_{y A} \\
i_{y B}
\end{array}\right] \\
&=-K_{s} q_{B}+K_{i} i,
\end{aligned}
$$

where $K_{s}$ is named magnetic stiffness matrix.

Therefore, the rotor dynamic equation turns to,

$$
\begin{aligned}
M \ddot{q}+G \dot{q}+B K_{s} q_{b} & =B K_{i} i, \\
y & =C q .
\end{aligned}
$$

And the vector $q_{b}$ can be replaced by $q$ through the identity $q_{b}=B^{T} q$ :

$$
q_{b}=\left[\begin{array}{l}
x_{b A} \\
x_{b B} \\
y_{b A} \\
y_{b B}
\end{array}\right]=\left[\begin{array}{llll}
a & 1 & 0 & 0 \\
b & 1 & 0 & 0 \\
0 & 0 & a & 1 \\
0 & 0 & b & 1
\end{array}\right]\left[\begin{array}{c}
\beta \\
x \\
-\alpha \\
y
\end{array}\right],
$$

Thus, the dynamics equation (6) is described coherently with the state vector adopted with the associated stiffness matrix $K s s$,

$$
M \ddot{q}+G \dot{q}+\underbrace{B K_{s} B^{T}}_{K_{s s}} q=B K_{i} i
$$

with,

$$
K_{s s}=\left[\begin{array}{cccc}
K_{11} & K_{12} & 0 & 0 \\
K_{21} & K_{22} & 0 & 0 \\
0 & 0 & K_{33} & K_{34} \\
0 & 0 & K_{43} & K_{44}
\end{array}\right]
$$


being,

$$
\begin{aligned}
& K_{11}=K_{33}=a^{2} k_{s A}+b^{2} k_{s B}, \\
& K_{12}=K_{21}=K_{34}=K_{43}=a^{2} k_{s A}+b^{2} k_{s B}, \\
& K_{22}=K_{44}=k_{s A}+k_{s B} .
\end{aligned}
$$

For the sensitivity analysis performed in this study, only the static case is concerned. Therefore, equations 7-8 become,

$$
K_{s s} q=B K_{i} i \text {. }
$$

Equation (9) is used in the next section to analyse the required control current from variations in three important model parameters.

\section{MAGNETIC STIFFNESS AND ITS EFFECTS ON THE CONTROL CURRENT}

Notice that $K_{s}$ carries parameters such as permeability, pole area, angle of the pole, air gap, base current and base winding. All of them are possible to be varied to change RMB's magnetic stiffness.

In this work, for constructive reasons, it is chosen to keep the angle between poles $\left(\alpha_{p}\right)$ and the base winding $\left(\omega_{0}\right)$ unchanged (air permeability $\mu_{0}$ is constant for nature). Hence, the main idea in this section is to verify the magnitude of the control current $i$ from a change in the remaining parameters, namely pole area $\left(A_{p}\right)$, base current $\left(i_{0}\right)$ and air gap $\left(s_{0}\right)$.

The analysis considers three different operating points:

1) $y=0$ (central equilibrium);

2) $y=-240 \mu \mathrm{m}$ (inferior equilibrium);

3) $y=+240 \mu \mathrm{m}$ (superior equilibrium).

Case 1) is the nominal one, whilst case 2) is the worst (more necessary current to win the gravitational force $g$ ) and case 3) requires possibly less control current since $g$ helps returning RMB's to nominal equilibrium.

Since it is difficult to visualise the impact each parameter in such a nonlinear function, the chosen method for the analyses is from a spectral cube, which allows the designer to observe four information at once. The three axis of the graph represent the variation of the pole area $\left(A_{p}\right)$, base current $\left(i_{0}\right)$ and air gap $\left(s_{0}\right)$, whereas the magnitude of the control current $i$ is represented by the change in colour of a small box positioned in each coordinate. The gradient of colour varies from blue (smaller values of $i$ ) to red (higher values of $i$ ).

Also for constructive reasons, the variation of the pole area $A_{p}$ is chosen to be between 5.18-36.2 $\mu^{2}$. The air gap varies within 336-624 $\mu \mathrm{m}^{2}$. On the other hand, the base current $i_{0}$ is clearly a design parameter. In this work it is defined that a suitable starting point for it is $2.5 \mathrm{~A}$, since it allows the control current to range \pm 2.5 A before switching to negative values. For the simulations, the base current ranges from 2.5-5.5 A.

Figure 5 shows the results for case 1 ). It is noted that the lowest control current $(i \approx 0.04 \mathrm{~A})$ occurs with the maximum pole area, maximum base current and minimum air gap. Conversely, the highest control current $(i \approx 2.03 \mathrm{~A})$ occurs with the minimum pole area, minimum base current and maximum air gap.

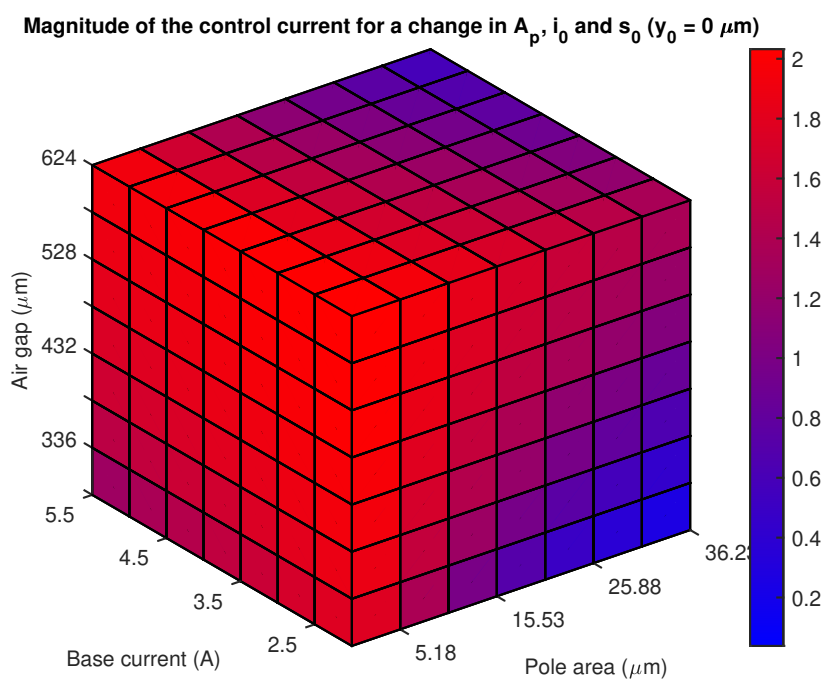

Fig. 5. Spectral cube for $y=0$.

The spectral cube presented in Fig. 6 shows the results for case 2 ). The lowest control current ( $i \approx 1.18 \mathrm{~A}$ ) occurs with the maximum pole area, minimum base current and maximum air gap. The highest control current ( $i \approx 3.9 \mathrm{~A}$ ) occurs with the minimum pole area, maximum base current and minimum air gap.

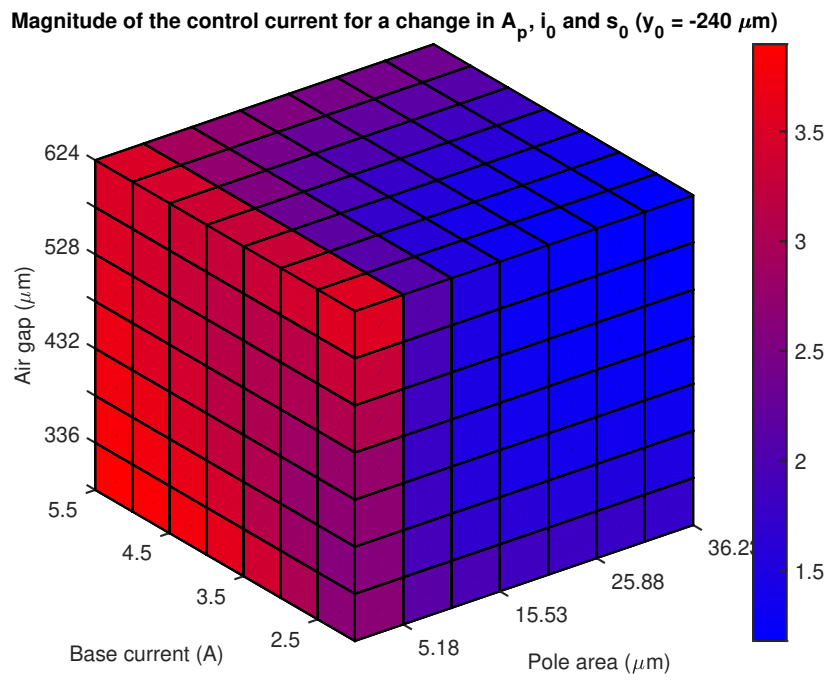

Fig. 6. Spectral cube for $y=-240 \mu \mathrm{m}$.

At last, Fig. 7 shows the results for case 3). The lowest control current $(i \approx-3.6 \mathrm{~A})$ occurs with the maximum pole area, maximum base current and minimum air gap. On the other had, the highest control current ( $i \approx 1.14 \mathrm{~A}$ ) occurs with the minimum pole area, minimum base current and maximum air gap. 


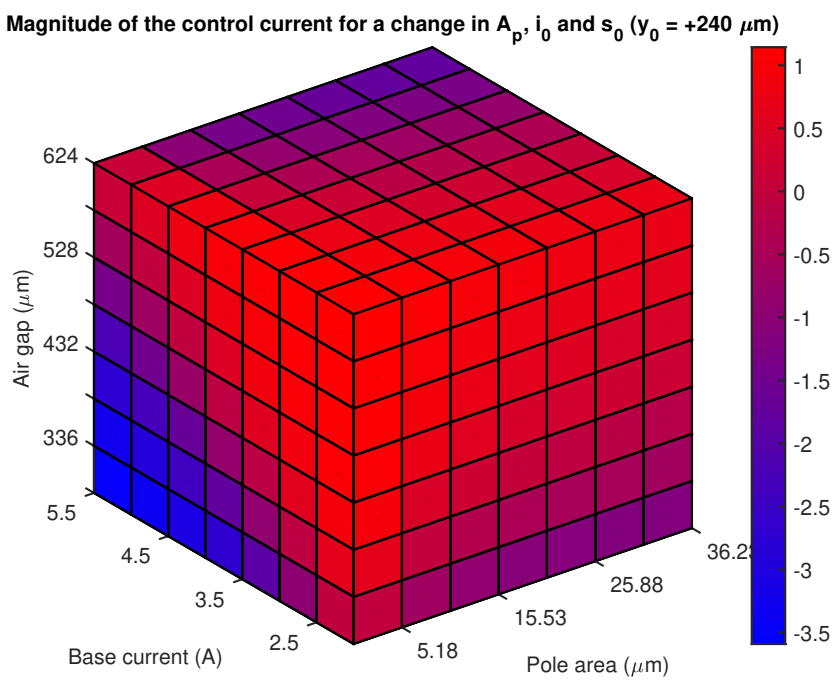

Fig. 7. Spectral cube for $y=+240 \mu \mathrm{m}$.

The next section presents the discussion of the results just obtained.

\section{DISCUSSION OF RESULTS}

It is important to start with the definition of what is required for a suitable project of the RMB. Having low values for the base and control currents is important aiming at energy consumption. However, it might not be the only issue to be concerned.

For instance, having a broader excursion of the control current is also interesting looking at the control point of view, since it increases the closed-loop sensitivity. At the same time, a low base current is also important to reduce RMB's energy consumption mainly at the central equilibrium point $(y=0)$. With these two information, one might say that the cube in Fig. 6 is the starting point of the design since it represents the worst case when one must compensate the gravity force.

For the cited figure, the highest control current $(i \approx 2.92$ A) with the lowest base current $i_{0}$ occurs with minimum pole area and maximum air gap. However, notice that this control current is higher than the base one. It is not recommended due to RMB's differential mode. Considering the base current $i_{0}$ as a limit for the control one, one finds the control current of $i \approx 2.5$ A with minimum pole area $\left(A_{p}=5.18 \mu m^{2}\right)$ and air gap of $s_{0}=528 \mu \mathrm{m}$. This result is taken as standard for the next analysis.

Regarding again $i_{0}=2.5 \mathrm{~A}, s_{0}=528 \mu \mathrm{m}$ and $A_{p}=5.18$ $\mu m^{2}$, for case 1) (Fig. 5), the control current must be $i \approx 1.45$ A, whilst for case 3) (Fig. 7) it is $i \approx 0.41 \mathrm{~A}$.

In fact, all three obtained results are theoretically suitable for use. However, a deeper analysis of RMB's magnetic saturation must also be performed. It is important since the control current is added to the base one, and the resulting current must have an amplitude that keeps the RMB relatively far from magnetic saturation. Considering the worst case (case 2) $-i_{0}=i=2.5$ A) for all windings, Fig. 8 shows the flux lines of the designed RMB, which indicates its saturation level and decoupling. Notice that with the specified currents the RMB keeps relatively far from magnetic saturation.
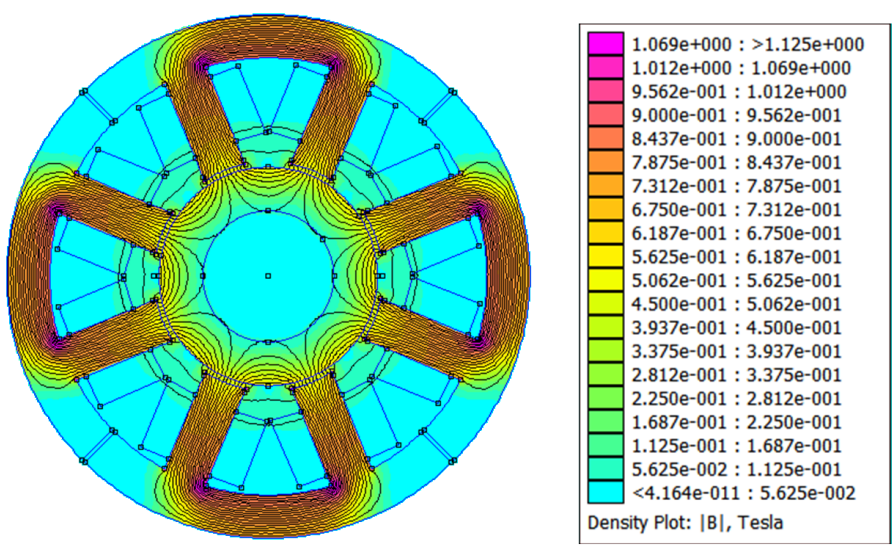

Fig. 8. Flux lines indicating saturation level of the RMB with specified parameters.

\section{CONCLUSION}

This paper has presented how some constructive parameters of a RMB might vary to change its magnetic stiffness, which is an important amount aiming at RMB's energy efficiency and further control.

Firstly the RMB dynamic modelling is presented in detail to show how to obtain the magnetic stiffness matrix $K_{s}$. Then, three parameters are varied $\left(A_{p}, s_{0}, i_{0}\right)$ in order to observe the control current magnitude. The results are presented using a spectral cube to ease the visualisation. Three simulations are performed: with the RMB at the central, inferior and superior positions.

From the analysis of those results, it is determined that the base current, pole area and air gap must have values of $i_{0}=$ $2.5 \mathrm{~A}, A_{p}=5.18 \mu \mathrm{m}^{2}$ and $s_{0}=528 \mu \mathrm{m}$, respectively. With these values, the control current has maximum magnitude of $i=2.5$ A (in the worst case with inferior position).

At last, a magnetic saturation analysis is also performed to verify whether these amounts keep the RMB far from saturation, maintaining it in the magnetic linear region.

The next steps of this work will deal with the closed-loop system and its relative control design of the RMB.

\section{ACKNOWLEDGMENT}

The authors would like to thank for the financial support of the Federal Institute of Santa Catarina (IFSC).

\section{REFERENCES}

[1] T. Yoshimoto, "Eddy current effect in a magnetic bearing model," IEEE Transactions on Magnetics, vol. 19, no. 5, pp. 2097-2099, 1983.

[2] B. Siciliano and O. Khatib, Springer handbook of robotics. springer, 2016.

[3] M. de Almeida Lopes, "Estudo de um mancal magnético eletrodinâmico," Ph.D. dissertation, Universidade Federal do Rio de Janeiro, 2014.

[4] D. Eaton, J. Rama, and S. Singhal, "Magnetic bearing applications \& economics," in 2010 Record of Conference Papers Industry Applications Society 57th Annual Petroleum and Chemical Industry Conference (PCIC). IEEE, 2010, pp. 1-9. 
[5] A. Storstenvik, "Subsea compression-designing and building a subsea compressor station," in Offshore Technology Conference. OnePetro, 2016.

[6] K. Laxminarayan, H. Jayanth, and M. V. Reddy, "Design and analysis of high-pressure casing of a steam turbine," Procedia Materials Science, vol. 5, pp. 1745-1754, 2014.

[7] L. A. Hawkins, B. T. Murphy, and J. Kajs, "Analysis and testing of a magnetic bearing energy storage flywheel with gain-scheduled, mimo control," in ASME Turbo Expo 2000: Power for Land, Sea, and Air. American Society of Mechanical Engineers Digital Collection, 2000.

[8] Y. Liu, S. Ming, S. Zhao, J. Han, and Y. Ma, "Research on automatic balance control of active magnetic bearing-rigid rotor system," Shock and Vibration, vol. 2019, 2019.

[9] Y. Zheng, N. Mo, Y. Zhou, and Z. Shi, "Unbalance compensation and automatic balance of active magnetic bearing rotor system by using iterative learning control," IEEE Access, vol. 7, pp. 122613-122625, 2019.

[10] N. Saeed and A. Kandil, "Lateral vibration control and stabilization of the quasiperiodic oscillations for rotor-active magnetic bearings system," Nonlinear dynamics, vol. 98, no. 2, pp. 1191-1218, 2019.

[11] A. Kandil, M. Sayed, and N. Saeed, "On the nonlinear dynamics of constant stiffness coefficients 16-pole rotor active magnetic bearings system," European Journal of Mechanics-A/Solids, vol. 84, p. 104051, 2020.

[12] J. A. Malagoli, "Análise da densidade de fluxo em um mancal magnético via método dos elementos finitos," in I Simpósio de Métodos Numéricos em Engenharia, 2016.

[13] W. Zhang and H. Zhu, "Radial magnetic bearings: An overview," Results in physics, vol. 7, pp. 3756-3766, 2017.

[14] T. Schuhmann, W. Hofmann, and R. Werner, "Improving operational performance of active magnetic bearings using kalman filter and state feedback control," IEEE Transactions on Industrial Electronics, vol. 59, no. 2, pp. 821-829, 2011.

[15] G. Schweitzer and E. H. Maslen, Magnetic bearings. theory, design, and application to rotating machinery, Springer, 2009. 